\title{
Dynamic tracking and mobility analysis of single GLUT4 storage vesicle in live 3T3-L1 cells
}

\author{
Chen Hong $\mathrm{LI}^{1, *}$, Li BAI ${ }^{1, *}$, Dong Dong $\mathrm{LI}^{1}$, Sheng $\mathrm{XIA}^{1}$, Tao XU ${ }^{1,2, * *}$ \\ ${ }^{1}$ Institute of Biophysics and Biochemistry, Huazhong University of Science and Technology, Wuhan 430074, China \\ ${ }^{2}$ National Key Laboratory of Biomacromoleculars, Institute of Biophysics, Chinese Academy of Sciences, Beijing 100101, \\ China
}

\begin{abstract}
Glucose transporter 4 (GLUT4) is responsible for insulin-stimulated glucose transporting into the insulin-sensitive fat and muscle cells. The dynamics of GLUT4 storage vesicles (GSVs) remains to be explored and it is unclear how GSVs are arranged based on their mobility. We examined this issue in 3T3-L1 cells via investigating the three-dimensional mobility of single GSV labeled with EGFP-fused GLUT4. A thin layer of cytosol right adjacent to the plasma membrane was illuminated and successively imaged at $5 \mathrm{~Hz}$ under a total internal reflection fluorescence microscope with a penetration depth of $136 \mathrm{~nm}$. Employing single particle tracking, the three-dimensional subpixel displacement of single GSV was tracked at a spatial precision of $22 \mathrm{~nm}$. Both the mean square displacement and the diffusion coefficient were calculated for each vesicle. Tracking results revealed that vesicles moved as if restricted within a cage that has a mean radius of $160 \mathrm{~nm}$, suggesting the presence of some intracellular tethering matrix. By constructing the histogram of the diffusion coefficients of GSVs, we observed a smooth distribution instead of the existence of distinct groups. The result indicates that GSVs are dynamically retained in a continuous and wide range of mobility rather than into separate classes.
\end{abstract}

Keywords: Insulin, GLUT4, GLUT4 storage vesicle (GSV), 3T3-L1, total internal reflection, fluorescence microscopy, single particle tracking.

\section{INTRODUCTION}

During glucose uptake, the glucose transporter 4 (GLUT4) forms an aqueous pore across the cell surface through which extracellular glucose can be transported to cytosol, a process that plays a crucial role in wholebody glucose homeostasis [1]. Accumulating evidence has suggested that irregularities in GLUT4 trafficking underlie type II diabetes [2-4]. GLUT4 is retained in GLUT4 storage vesicles (GSVs) that dynamically recycle among many intracellular organelles, including the plasma membrane, sorting endosomes, recycling endosomes and trans-Golgi network (TGN) $[1,5,6]$.

\footnotetext{
"These authors contributed equally to this work.

${ }^{* *}$ Correspondence: Tao XU

E-mail: txu@mail.hust.edu.cn

Abbreviations: GLUT4, glucose transporter; GSVs, GLUT4 storage vesicles; SPT, single particle tracking; TIRFM, total internal reflection fluorescence microscopy; EGFP, enhanced green fluorescence protein; SNARE, soluble N-ethylmaleimide-sensitive factor attachment protein receptor.
}

Most of the current knowledge about the GSV trafficking pathway is indirectly obtained by biochemical approaches and the measurement of steady-state information. By fluorescence quenching assay combined with chimeric reporters, it was indicated that GLUT4 recycles through the transferring-receptor-containing endosomal recycling compartment, whose reversible retention controls the levels of the proteins on cell surface [7-9]. Besides, a fraction of GLUT4 moves through endosomes into TGN and this pathway is mediated by an unique acidic amino acid in the carboxyl terminus of GLUT4 [10-13]. GSVs contain the so-called v-SNARE (soluble Nethylmaleimide-sensitive factor attachment protein receptor), vesicle associated membrane protein (VAMP2), suggesting GLUT4 trafficking and fusion share similarities with other regulated membrane fusion processes [14-16]. It seems likely that various recycling loops must be coordinated in regulating the trafficking of intracellular GLUT4. Although different GLUT4 trafficking pathways have been proposed, it is still unclear whether GSVs are classified into distinct fractions according to their mobility. 
It would clearly be helpful to investigate the mobility of a single GSV in live cells. Here we have labeled the GSVs by constructing a fusion protein that links EGFP to the Cterminus of mouse GLUT4. The chimeric GLUT4-EGFP fusion protein was expressed in 3T3-L1 cells by transient transfection. By total internal reflection fluorescence microscopy (TIRFM), a thin layer of cytosol immediately adjacent to the cytosol-coverslip interface was selectively illuminated and the corresponding fluorescence images were recorded with charge coupled device (CCD) at high time resolution. In order to obtain the sub-pixel displacement of GSVs, we employed a Gaussian-based single particle tracking (SPT) method to resolve the kinetics of vesicle motion. We found that GSVs move in a constrained fashion as if tethered by some intracellular structure. The distribution of the three-dimensional (3D) diffusion coefficients $\left(D^{(3)}\right)$ was a smooth continuum. Our results demonstrate that despite the existence of different recycling loops for GSVs, they are organized in a continuous range of mobility rather than into separate classes.

\section{MATERIALS AND METHODS}

\section{GLUT4-EGFP construction and cell culture}

To construct EGFP-tagged GLUT4, the open reading frame (ORF) of GLUT4 was amplified by polymerase chain reaction (PCR) with pfu DNA polymerase (Stratagen, La Jolla, CA) from mice genomic DNA which was isolated from mice tail with DNeasy Tissue Kit (Qiagen, Hilden, Germany). The sequences of GLUT4 primers are: Forward 5'TACCGCTCGAGACAAGATGCCGTCGGGTTTCCAGCAGATCG3'. Reverse primer: 5'-GCTAGGATCCCCGTCATTCTCATCTGGCCCTAAGTATTCAAG-3'. The amplified PCR products were cloned in frame into the EcoRI and BamHI sites of the mammalian expression vector pEGFP-N1 (Clontech Laboratories, Palo Alto, CA).

3T3-L1 cells were cultured in DMEM (Gibco Inc.) supplied with $10 \%$ new calf serum (Gibco Inc.). Then, cells were plated in chambers mounting ploy-l-lysine coated coverslips and were transfected when cells grew to $80 \%$ confluence by lipofectamine 2000 (Invitrogen, Inc.) according to the manufacturer's instruction. Ten hous after transfection, the cell culture medium was replaced with serum-free DMEM, and was viewed $2 \mathrm{~h}$ later under TIRFM.

\section{Microscopy}

To confirm the intracellular distribution of GLUT4 throughout the cell, transfected live 3T3-L1 cells were viewed with a confocal laser scanning biological microscope (FV500, Olympus Inc, Japan) with a-plan Fluar NA 1.45 objective ( $\times 100$, Zeiss Inc., Germany). Images were acquired and analysed using Fluoview software that came along with the confocal microscope.

To selectively excite a thin layer of cytosol immediately adjacent to the coverslip, we used a TIRFM constructed with an inverted microscope (IX-70, Olympus) equipped with a NA 1.65 objective (Apo $\times 100$, OHR, Olympus). Immersion oil (refractive index $=1.81$, Cargille Laboratories Inc, USA) was used to bridge the optical contact between the objective and the coverslip. Light from an argon ion laser (Melles Griot Inc, USA) was passed through a shutter which is controlled by image acquisition software (TILL vision 4.0; Till Photonics GmBH, Germany). The shutter was opened only during camera exposure. The light was guided to a KineFLEX singlemode fiber (Point Source Co, UK). The angle of the incident laser was adjusted to give an evanescent field with a penetration depth of 136 $\mathrm{nm}$.

Emission fluorescence was selected with a GFP filter set (Chroma, USA) prior to being recorded by a cooled CCD (PCO SensiCam; Germany), which has a pixel size of $67 \mathrm{~nm} \times 67 \mathrm{~nm}$ at the specimen plane. The focal plane was precisely adjusted via moving the objective with a piezoelectric $z$-axis controller (E-662. LR; Physik Instruments, Germany). During imaging period, the extracellular medium was superfused with the buffer containing (in $\mathrm{mM}$ ) 125 $\mathrm{NaCl}, 5 \mathrm{KCl}, 1.3 \mathrm{CaCl}_{2}, 1.2 \mathrm{MgSO}_{4}, 20$ D-Glucose, 25 Hepes (pH 7.4). We collected the images of 3T3-L1 cells at $5 \mathrm{~Hz}$ with the exposure time of $50 \mathrm{~ms}$. All experiments were performed at room temperature.

\section{Tracking single GSV}

We have employed a Gaussian-based SPT method, which has been proved as the most effective approach in tracking single particles [17]. Briefly, averaged extracellular background was subtracted from the images prior to tracking. By fitting Gaussian equation to the fluorescence intensity distribution of single vesicles, we could obtain the peak position that correspond the lateral position coordinates $(x, y)$, peak intensity $(F)$, full width at half-maximal (FWHM) intensity, and the local background $(b)$. The lateral displacement of single vesicles could thus be obtained by estimating the change in position among consecutive images. This way we have successfully resolved the sub-pixel displacement of fluorescence labeled particles from consecutive images [18-20].

According to the exponential relationship between the fluorescence intensity of a particle and its vertical position as this particle moves within evanescent field, the vertical position of the particle can be obtained from the changes in vesicle's fluorescence intensity [21-23]. Here we calculated vertical displacement as

$$
z_{n}=-d \ln \left(F_{n-1}-B_{n-1}\right) /\left(F_{n}-B_{n}\right)
$$

where $d$ is the penetration depth, $F n$ and $B n$ are the peak intensity and local background in frame $n$, respectively.

The mean square displacement (MSD) for each vesicle was calculated as follows

$$
M S D(t)=\frac{1}{L-n} \sum_{s=0}^{L-n-1}(r(s+n)-r(s))^{2}
$$

where $n=t / \Delta t, \Delta t$ is the time interval between two successive TIRFM-recorded images, $L$ is the total number of images in a recorded sequence, and $r(s)$ is the 3D position of vesicle in frame $s$.

\section{Statistics}

Population averages were expressed as mean \pm SEM. The median and median standard error (MSE) were used to describe skewed distributed data.

\section{RESULTS}

\section{Imaging single GSVs}

It has been previously reported that most of the GLUT4 are localized in the cytosol in resting 3T3-L1 cells using immunofluorescence imaging $[1,6]$. In order to gain insight into the spatial distribution and dynamics of GLUT4 
in live cells, we tagged the GLUT4 protein with a green fluorescence protein (GFP). By transfecting with the fusion protein, we would be able to determine the intracellular localization and track the motion of GLUT4 in live cells using either the confocal microscopy or TIRFM. Confocal microscopy offers the advantage of improved spatial resolution due to its ability to reduce most of the out-of-focus fluorescence. When the chimeric GLUT4EGFP was expressed in 3T3-L1 cells, we observed a punctate distribution of the fluorescence under confocal laser scanning microscope (Fig. 1A). The result is consistent with the sequestration of GLUT4 to GSVs and most of the GLUT4 is retained inside unstimulated cells. However, due to the rapid photobleaching and phototoxicity, confocal microscopy is not suitable for long-term imaging of live cells [24]. The rather slow imaging rate of confocal microscopy further limits its application in dynamic tracking which requires high time resolution. So we turned to TIRFM for real-time tracking of GLUT4. As shown in Fig. 1B, GLUT4 also appeared as distinct fluorescence spots under TIRFM. These fluorescence spots were not static but rather in rapid movement.

Next we tested whether the EGFP-labeled organelles were functional competent for insulin-dependent insertion into the plasma membrane. The evanescent field, generated by the total reflection, declines exponentially with the distance from the glass-water interface. Thus, if a fluorescent particle approaches the basal plasma membrane adhere to the coverglass, as if happens during fusion, one could observe a brightening of the fluorescence followed by lateral diffusion of the fluorescence in the membrane. We challenged the cells with $200 \mathrm{nM}$ insulin and looked for insulin-dependent fusion of fluorescent organelles. To judge for fusion events, we placed two concentric circles around the vesicle center, with the small circle just large enough to accommodate the image of the vesicle (Fig. 2B). The difference in fluorescence intensities between the inner circle and the surrounding annulus serves as the resulted fluorescence intensity and is plotted against time. The resulted fluorescence from one spot is shown in Fig. $2 \mathrm{C}$ where it increases at first, declines after a peak and finally fluctuates near the zero level. This pattern is consistent with the full fusion of secretory granules. The sequential images also display the diffusion and disappearance of fluorescence (Fig. 2A). Thus, the fluorescence spots are likely representing the GSVs, where EGFPlabeled GLUT4 is stored and able to undergo insulinstimulated insertion into the plasma membrane.

\section{Fluctuation in fluorescence spots reflects the vertical movement of GSVs}

Due to the exponential decay of the evanescent field, the fluorescence intensity of GSVs is proportional to their
A

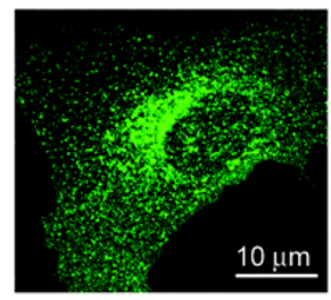

B

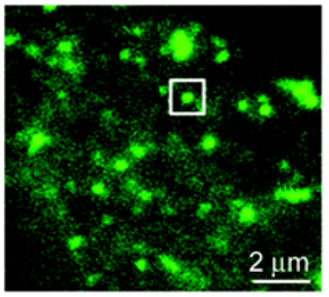

Fig. 1 Subcellular distribution of EGFP-labeled GLUT4 in 3T3-L1 cells. (A) Representative fluorescence image of the cells expressing GLUT4-EGFP under confocal laser scanning microscope. (B) Evanescent-field micrograph of the footprint of a cell transiently transfected with GLUT4-EGFP under TIRFM. The cells were transiently transfected with plasmids encoding GLUT4-EGFP one night before the experiment. Both techniques reveal punctate distribution of the fluorescence, suggesting EGFP-labeled GLUT4 is sequestered into intracellular organelles.

A

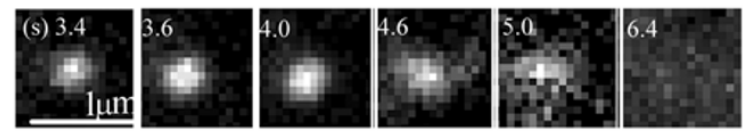

B

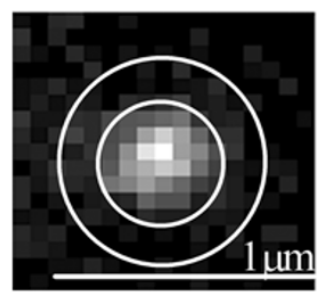

C

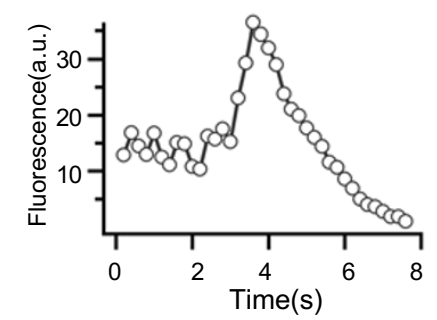

Fig. 2 Vesicles containing GLUT4-EGFP are competent for insulinstimulated insertion into the plasma membrane. 3T3-L1 cells expressing GLUT4-EGFP were stimulated with $200 \mathrm{nM}$ insulin extracellularly, and viewed under TIRFM. (A) Sequential images of a single vesicle labeled with GLUT4-EGFP undergoing fusion with the plasma membrane. (B) A vesicle surrounded by two concentric circles $(0.47 \mu \mathrm{m}$ and $0.74 \mu \mathrm{m}$ in diameter $)$ defining a circle and annulus for the measurement of radial gradients in fluorescence. (C) The difference in fluorescence intensities between the circle and annulus (circle minus annulus) is plotted against time. The brief, spike-like fluorescence increase marks fusion. The decline of the difference reflects the diffusion of GLUT4 in the membrane.

axial position to the cell surface $[22,25,26]$. In Fig. 3A, we showed the fluctuation of the fluorescence of a single GSV. As you can see, this GSV repetitively brightened and dimmed within a period of $10 \mathrm{~s}$. We calculated the mean intensity of a $0.871 \mu \mathrm{m} \times 0.871 \mu \mathrm{m}$ square that was centered on the vesicle and displayed the time course of the fluorescence intensity in Fig. 3B. Since the fluorescence 

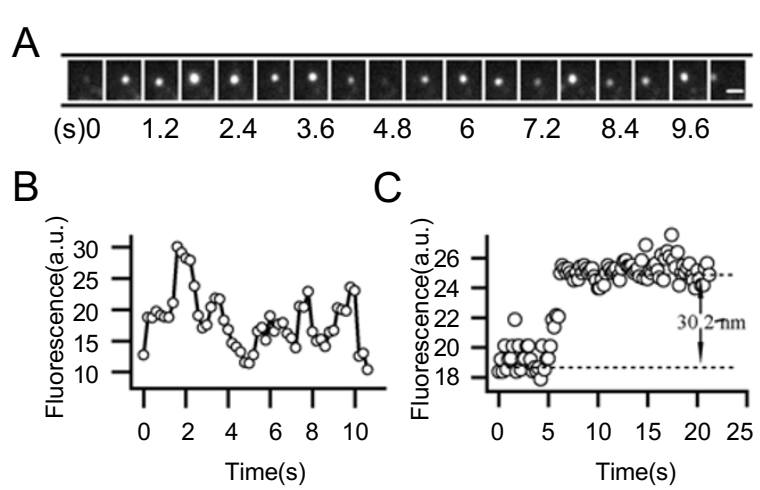

Fig. 3 Fluorescence fluctuation reflects the movement of vesicles towards or away from the plasma membrane. (A) Sequential images of a GSV (marked in the box in Fig. 1B) approaching the plasma membrane several times. Scale bar, $1 \mu \mathrm{m}$. (B) Plot of the fluorescence change of the vesicle shown in (A) as a function of time. The vesicle became abruptly bright during the first seconds, reflecting a sudden approach to the cell surface, and then the fluorescence rapidly declined and brightened several times. (C) An example of one vesicle jumping from a stationary position to another. Two plateaus reflecting a vertical distance of $30.2 \mathrm{~nm}$ are clearly distinguished.

A

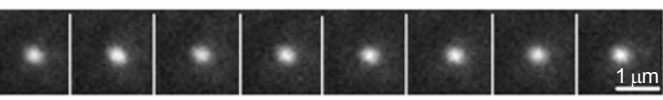

(s) $0 \quad 4$

6

C
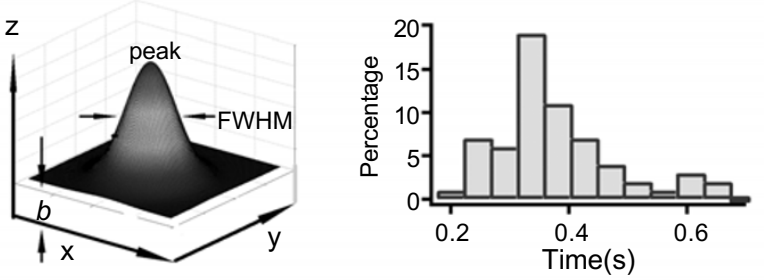

Fig. 4 Size distribution of GSVs. (A) Sequential images of a vesicle slightly moving around a fixed position. (B) The fluorescence of a single GSV displays a typical Gaussian distribution. We fit the fluorescence of each GSV with a two-dimensional Gaussian function plus a local background constant (marked as " $b$ "). According to the fit, the peak of the fluorescence and the full width at half maximum (FWHM) are determined. (C) Histogram distribution of FWHM from the Gaussian fits to 90 individual GSVs. The averaged FWHM of GSVs is $0.36 \pm 0.02 \mu \mathrm{m}$.

intensity is dependent on the axial position of vesicles, the fluorescence fluctuation reflects the vertical movement of this vesicle towards or away from the plasma membrane. Upon the appearance of GSV in the evanescent field, its fluorescence will abruptly become brighter. When the GSV is retreated away from the plasma membrane, its fluorescence will also decline. Most of the GSVs displayed multiple up and down of their fluorescence intensity as shown in Fig. $2 \mathrm{~B}$, reflecting the repetitive approaching and departure of GSVs in relative to the plasma membrane. The rapid transition of GSVs relating to the plasma membrane also suggests that most of the GSVs are not docked to the plasma membrane. Occasionally, we observed an abrupt increase of fluorescence in a single GSV and then the fluorescence remained constant for a fairly long time (Fig. 3C), indicating the jump of vesicle from one axial position to another. According to the fluorescence change and the measured penetration depth, the distance between the two plateaus residence by this vesicle is about $30.2 \mathrm{~nm}$. The jump suggests that some kinds of structures may help the vesicle to move along axial direction.

\section{D sub-pixel displacement of single GSVs revealed by SPT}

The rationale of sub-pixel displacement detection is that the fluorescence of single particle spreads over more than one pixel in recorded images, so the sub-pixel displacement can be tracked by weighting the fluorescence distribution from multiple pixels between successive time-lapse images (Fig. 4A). Gaussian-based tracking method is the superior algorithm in terms of both accuracy and precision, and is most robust to imaging noise [17]. So, we directly fitted Gaussian equation to the intensity distribution of the single vesicle, thereby yielding the lateral position and peak intensity (Fig. 4B). The lateral displacement of individual GSV was obtained by comparing the vesicle position among successive images, and the axial distance moved by a vesicle could be calculated from the fluctuation in peak intensity. Because the Gaussian-based SPT method become less reliable when the signal-to-noise ratio (SNR) is less than 4 [17], we chose those vesicles with SNR more than 6 to ensure the precision of tracking. Next, we estimated the size of the single GSV by measuring the full width at half maximum (FWHM) fluorescence. The FWHM was estimated to be around $0.36 \pm 0.02 \mu \mathrm{m}$ (Fig. 4C, $n=90$ ), very similar to the size of $0.38 \mu \mathrm{m}$ measured for large dense core vesicles in PC12 cells [25, 27].

\section{Restriction of the motion of GSVs}

The 3D trajectory of one GSV tracked by our SPT method was constructed in Fig. 5A. Our tracking result shows that all the 3D displacements traveled by this vesicle are less than $100 \mathrm{~nm}$, indicating the wandering of this vesicle around its initial site. From the calculation of MSD, one can tell the different motion types of GSVs [23, 28]. The averaged MSD was plotted against time in Fig. 5B. The negative curvature observed in the MSD plot manifests a caged diffusion for GSVs, which means that the 


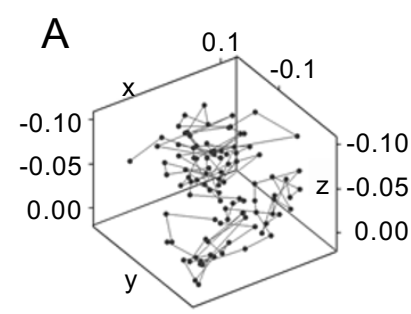

Trajectory $(\mu \mathrm{m})$
B

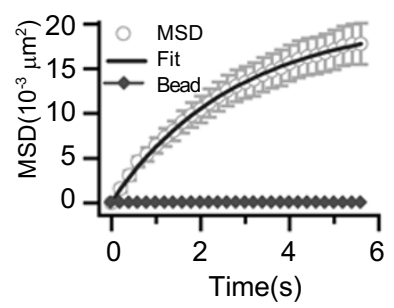

Fig. 5 Constrained motion of GSVs underneath the plasma membrane. (A) 3D trajectory of one GSV constructed using single particle tracking method as explained in the Materials and Methods section. The displacements travelled by the vesicle in three axis are less than 100 $\mathrm{nm}$, indicating the wandering of this vesicle around its initial site. (B) Averaged MSD $\pm \mathrm{SE}$ (Grey circles) of 129 individual GSV trajectories from 13 cells recorded at 5 frames/s. The line is the best leastsquares fit of Equation 3. The fit parameters are given in the text. The averaged MSD displays a negative curvature, suggesting a constrained diffusion of GSV within a cage of $160 \mathrm{~nm}$ in radius. As a comparison, the averaged MSD of immobilized beads is displayed, which reflects the lower limit of our detection in motion tracking.

vesicle motion is confined in some cage-like tether constructed by neighboring obstruction. This feature can be described with the following approximate equation [29]:

$$
M S D(n \Delta t)=R^{2}\left[1-A_{1} \exp \left(-6 A_{2} D^{(3)} n \Delta t / R^{2}\right)\right]
$$

where $R$ is the radius of the spherical cage in which particle is free to diffuse. Indeed, the averaged MSD plot was well fitted with this equation (grey continuous line in Fig. 5B). The mean radius of cage tethering GSVs was estimated to be $0.16 \pm 0.01 \mu \mathrm{m}(n=129)$. The wondering of GSV inside the cage was not due to the drift of our recording system, because the immobilized beads $(0.175$ $\mu \mathrm{m}$ in diameter, Molecular Probes) displayed little movement (Fig. 5B). The $D^{(3)}$ of immobilized beads was estimated to be $0.04 \times 10^{-4} \mu \mathrm{m}^{2} / \mathrm{s}$, which reflects the lower limit of our detection with a spatial precision of $22 \mathrm{~nm}$ for SPT.

\section{Smooth continuum of $D^{(3)}$ among GSVs}

In muscle and fat cells, GLUT4 is sorted, retained and recycled by different trafficking pathways with special molecular mechanism [1]. However, whether and how GSVs are coordinated according to their mobility is still unclear. Measuring the $D^{(3)}$ of individual GSV by combining TIRFM and SPT technology, we answer this question via constructing the histogram of $D^{(3)}$ to reflect the distribution of GSVs based on their mobility. Our result shows that the $D^{(3)}$ of GSVs is distributed within a smooth and skewed continuum rather than distinct classes (Fig. 6A). The median of $D^{(3)}$ was calculated to be $(18.6 \pm 7.6) \times 10^{-4} \mu \mathrm{m}^{2} / \mathrm{s}$.
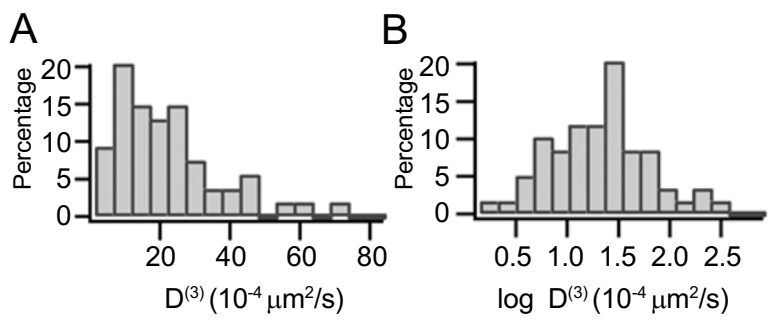

Fig. 6 Distribution of the 3D diffusion coefficients $\left(D^{(3)}\right)$ of GSVs. The diffusion coefficients are deduced from SPT of individual GSVs $(\mathrm{n}=129)$ performed with TIRFM. (A) Histogram distribution of $D$ (3) using linear binning displays a markedly skewed distribution. (B) Histogram of $D^{(3)}$ using logarithmic binning also displays a broad distribution. No clear separation in mobility of GSVs is observed.

Because of the limited time points measured in SPT performance, multiple pools of vesicles differing in their mobility may be hided in a broad and smooth distribution of diffusion coefficient [30]. However, when the abscissa of the distribution is displayed in a logrithmic scale, i.e., the whole distribution is shown in a semilogrithmic plot, separated classes could be clearly discernible [31]. So, as a further check, we displayed the histogram of $D^{(3)}$ in semilogarithmic presentation. The result is still a smooth continuum and is not evident with the existence of separated states of mobility (Fig. 6B).

\section{DISCUSSION}

In this study, we have labeled the GSVs with EGFPfused GLUT4 in pre-adipocytes 3T3-L1. Combining livecell confocal microscopy, TIRF microscopy and single particle tracking method, we have explored the spatial distribution and 3D mobility of GSVs in intact 3T3-L1 cells. Our results help to understand the dynamics of the movement of GSVs inside the cell and to clarify how GSVs are arranged based on their mobility.

Under confocal microscopy, we observed that the GLUT4-EGFP fluorescence exhibited punctuate spots throughout the cytosol, which is consistent with the vesicular localization of GLUT4 and that most of the GLUT4 is localized in the cytosolic compartments in unstimulated cells. This distribution agrees with previous reports using steady-state immunofluorescence method on fixed cells $[1,6,32]$. In addition, we found that the fluorescence spots are mobile rather than fixed under TIRFM, further confirming the labeling of GSVs.

The advantages of low background, high temporal resolution, minimal photobleaching and phototoxicity have made TIRFM very popular in long-term tracking of single vesicles in live cells $[23,25,26]$. SPT can be applied to 
resolve sub-pixel displacement of fluorescence labeled particles[18-20]. In this study, sub-pixel 3D tracking of the single GSV shows a clearly constrained motion. This kind of constrained (or caged) diffusion has been also suggested for secretory granules in the vicinity of the plasma membrane $[23,25,33]$, which is seen as the entrapment of granules inside the cortical actin cytoskeleton meshwork. In fact, granule trafficking is likely to be mediated by different cytoskeleton systems along with a number of motor proteins $[34,35]$. So, we interpret the restricted motion of GSVs as being constrained by nearmembrane actin cortex or other heterogeneous organelleconstructed matrices. The similarity of the size of GSVs with that obtained for secretory granules and a larger cage size estimated by our experiment than that of $70 \mathrm{~nm}$ observed for secretory granules [25] suggest GSVs are associated to a looser matrix in $3 \mathrm{~T} 3-\mathrm{L} 1$ cells.

The diffusion coefficient calculated for GSVs is $18.6 \times$ $10^{-4} \mu \mathrm{m}^{2} / \mathrm{s}$, which is consistent with that of $19 \times 10^{-4} \mu \mathrm{m}^{2} / \mathrm{s}$ measured for secretory granules in chromaffin cells with TIRFM [25]. It has been proposed that GSV contains SNARE-associated proteins that were initially found in secretory granules $[15,16]$. Indeed, the intracellular transport of secretory granules is selectively modulated by these proteins [14]. Thus, our finding of the similarity of diffusion coefficient between secretory granules and GSVs suggests that their motion might be regulated by a similar mechanism in the vicinity of the plasma membrane.

The spatial distribution and distinct trafficking pathways of GLUT4 among many organelles have been identified by the steady-state study [5, 7-9]. But it is not demonstrated whether GSVs are distributed into distinct pools based on their mobility. By examining the 3D mobility of individual GSV in live cells, our study reports a smooth continuum of diffusion coefficient rather than distinct groups of mobility. This finding demonstrates that there are no separate states of mobility for GSVs, instead GSVs are organized into a wide range of mobility. The smooth histogram of $D^{(3)}$ of GSVs differs significantly from a Gaussian distribution. It has long been presumed that vesicular exocytosis is limited because of the existence of distinct pools of releasable and refractory vesicles [36]. It was proposed that the refractory pool is a distinct immobile fraction of cytoplasmic vesicles [37]. Yet, we found the distribution of GSVs diffusion coefficients is broad and asymmetric rather than the presence of distinct pools or fractions. Recently, it has been suggested that the broad distribution of vesicles would affect the time course of vesicular release [31]. Thus, the delivery of GLUT4 to the plasma membrane is likely regulated by the unusual variation of the diffusion constants of GSVs, rather than the existence of separated fractions of vesicles with distinct mobility.

Employing advanced time-lapse fluorescence microscopy and fluorescent protein-tagged chimaeras has facilitated our study of the intracellular movement of GSVs. Further work will be needed to elucidate how GLUT4 is transported from one organelle to another, what regulatory mechanisms control the process, and which molecules are required.

\section{ACKNOWLEGEMENTS}

We thank Dr. Kan LIAO from the Shanghai Institute of Biological Sciences, Chinese Academy of Siences for providing the 3T3-L1 cells; Shang Bang GAO and Xiang Ping XU for the assistance in cell preparation. This work was supported by National Natural Science Foundation of China Nos. 30025023,3000062 and 30130230 to Tao XU, and Major State Basic Research Development Program of China (G1999054000 and 2004CB720000) to Tao XU. We are grateful for the support from Hubei Science Foundation Grants No. 2003ABA096 to Chen Hong LI.

Received, Aug 6, 2004

Revised, Oct 22, 2004

Accepted, Nov 8, 2004

\section{REFERENCES}

1 Bryant NJ, Govers R, James D. Regulated transport of the glucose transporter glut4. Nat Rev Mol Cell Biol 2002; 3: 267-77

2 Petersen KF, Shulman GI. Pathogenesis of skeletal muscle insulin resistance in type 2 diabetes mellitus. Am J Cardiol 2002; 90: $11 \mathrm{G}-18 \mathrm{G}$..

3 Hirohito Sone, Hiroaki Suzuki, Akimitsu Takahashi and Nobuhiro Yamada Disease model: hyperinsulinemia and insulin resistance. TRENDS in Molecular Medicine 2001; 7:320-2.

4 Garvey WT. Glucose transport and NIDDM.Diabetes Care 1992; 15:396-417.

5 Chiang SH, Baumann CA, Kanzaki M, et al. Insulin-stimulated GLUT4 translocation requires the CAP-dependent activation of TC10. Nature 2001; 410:944-8

6 Bogan JS, Hendon N, McKee AE, Tsao TS, Lodish HF. Functional cloning of TUG as a regulator of GLUT4 glucose transporter trafficking. Nature 2003; 425:727-34

7 Lampson MA, Schmoranzer J, Zeigerer A, Simon SM, McGraw TE. Insulin-regulated Release from the Endosomal Recycling Compartment Is Regulated by Budding of Specialized Vesicles. Mol Biol Cell 2001; 12:3489-501

8 Zeigerer A, Lampson MA, Karylowski O, et al. GLUT4 retention in adipocytes requires two intracellular insulin-regulated transport steps. Mol Biol Cell 2002; 13:2421-35

9 Karylowski O, Zeigerer A, Cohen A, McGraw TE. GLUT4 is retained by an intracellular cycle of vesicle formation and fusion with endosomes. Mol Biol Cell 2004; 15:870-82

10 Molloy SS, Anderson ED, Jean F, Thomas G. Bi-cycling the furin pathway: from TGN localization to pathogen activation and embryogenesis. Trends Cell Biol 1999; 9:28-35 
11 Xiang Y, Molloy SS, Thomas L, Thomas G. The PC6B cytoplasmic domain contains two acidic clusters that direct sorting to distinct trans-Golgi network/endosomal compartments. Mol Biol Cell 2000; 11:1257-73

12 Martin S, Ramm G, Lyttle CT, et al. Biogenesis of insulinresponsive GLUT4 vesicles is independent of brefeldin A-sensitive trafficking. Traffic 2000; 1:652-60

13 Palacios S, Lalioti V, Martinez-Arca S, Chattopadhyay S, Sandoval IV. Recycling of the insulin-sensitive glucose transporter GLUT4. Access of surface internalized GLUT4 molecules to the perinuclear storage compartment is mediated by the Phe5Gln6-Gln7-Ile8 motif. J Biol Chem 2001; 276:3371-83

14 Jahn R, Lang T, Südhof TC. Membrane Fusion. Cell 2003; 112: 519-33.

15 Hashiramoto M, James DE. Characterization of insulin-responsive Glut4 vesicles isolated from 3T3-L1 adipocytes. Mol Cell Biol 2000; 20:416-427

16 Ramm G, Slot JW, James DE, Stoorvogel W. Insulin recruits GLUT4 from specialized VAMP2-carrying vesicles as well as from the dynamic endosomal/trans-Golgi network in rat adipocytes. Mol Biol Cell 2000; 11:4079-91

17 Cheezum MK, Walker WF, Guilford WH. Quantitative comparison of algorithms for tracking single fluorescent particles. Biophys J 2001; 81:2378-88

18 Kues T, Dickmanns A, Luhrmann R, Peters R, Kubitscheck U. High intranuclear mobility and dynamic clustering of the splicing factor U1 snRNP observed by single particle tracking. Proc Natl Acad Sci U S A 2001; 98:12021-6

19 Goulian M, Simon SM. Tracking single proteins within cells. Biophys J 2000; 79:2188-98

20 Thompson RE, Larson DR, Webb WW. Precise nanometer localization analysis for individual fluorescent probes. Biophys J 2002; 82:2775-83

21 Ölveczky BP, Periasamy N, Verkman AS. Mapping fluorophore distributions in three dimensions by quantitative multiple angletotal internal reflection fluorescence microscopy. Biophys J 1997; 73:2836-47

22 Steyer JA, Almers W. A real-time view of life within $100 \mathrm{~nm}$ of the plasma membrane. Nat Rev Mol Cell Biol, 2001; 2:268-75
23 Oheim M, Stühmer M. Tracking chromaffin granules on their way through the actin cortex. Eur Biophys J, 2000; 29:67-89

24 Rizzuto R, Carrington W, Tuft RA. Digital imaging microscopy of living cells. Trends Cell Biol 1998; 8:288-92

25 Steyer JA, Almers W. Tracking single secretory granules in live chromaffin cells by evanescent-field fluorescence microscopy. Biophys J 1999; 76:2262-71

26 Becherer U, Moser T, Stuhmer W, Oheim M. Calcium regulates exocytosis at the level of single vesicles. Nat Neurosci 2003, 6: 846-53.

27 Lang T, Wacker I, Wunderlich I, et al. Role of actin cortex in the subplasmalemmal transport of secretory granules in PC-12 cells. Biophys J 2000; 78:2863-77

28 Qian H, Sheetz MP, Elson EL. Single particle tracking. Analysis of diffusion and flow in two-dimensional systems. Biophys J 1991; 60:910-21

29 Saxton MJ, Jacobson K. Single-particle tracking: applications to membrane dynamics. Annu Rev Biophys Biomol Struct 1997; 26:373-99

30 Saxton MJ. Single-particle tracking: the distribution of diffusion coefficients. Biophys J 1997; 72:1744-53

$31 \mathrm{Ng} \mathrm{YK}, \mathrm{Lu}$ X, Gulacsi A, et al. Unexpected mobility variation among individual secretory vesicles produces an apparent refractory neuropeptide pool. Biophys J 2003; 84:4127-34

32 Liu LB, Omata W, Kojima I, Shibata H. Insulin recruits GLUT4 from distinct compartments via distinct traffic pathways with differential microtubule dependence in rat adipocytes. J Biol Chem 2003; 278:30157-69

33 Johns LM, Levitan ES, Shelden EA, Holz RW, Axelrod D. Restriction of secretory granule motion near the plasma membrane of chromaffin cells. J Cell Biol 2001; 153(4):177-90

34 Hirokawa N. Kinesin and dynein superfamily proteins and the mechanism of organelle transport. Science 1998; 279:519-26

35 Kamal A, Goldstein LS. Principles of cargo attachment to cytoplasmic motor proteins. Curr Opin Cell Biol 2002; 14:63-8

36 Thorn, NA. In vitro studies of the release mechanism for vasopressin in rats. Acta Endocrinol 1966; 53:644-54.

37 Burke NV, Han W, Li D, et al. Neuronal peptide release is limited by secretory granule mobility. Neuron 1997;19:1095-102. 